\title{
Antibunched $N$-photon bundles emitted by a Josephson photonic device
}

\author{
Sheng-li Ma, ${ }^{*}$ Xin-ke Li, ${ }^{*}$ Ya-long Ren, Ji-kun Xie, and Fu-li $\mathrm{Li}^{\dagger}$ \\ MOE Key Laboratory for Nonequilibrium Synthesis and Modulation of Condensed Matter, Shaanxi Province Key Laboratory of Quantum \\ Information and Quantum Optoelectronic Devices, School of Physics, Xi'an Jiaotong University, Xi'an 710049, China
}

(Received 19 April 2021; revised 15 May 2021; accepted 22 September 2021; published 7 October 2021)

\begin{abstract}
We propose an experimentally feasible scheme for generating antibunched $N$-photon bundles by a dc voltage-biased Josephson junction in series with a superconducting microwave resonator and a charge qubit. Each resonant tunneling Cooper pair leads to the excitation of the charge qubit and resonator with $N$ photons simultaneously via the ac Josephson effect. Meanwhile, the charge qubit with strong anharmonicity is utilized to regulate the tunneling behavior of the Cooper pairs; that is, the presence of $N$ photons in the resonator prevents the next tunnel event. So the resonator contains only $N$ photons or none, and all other possibilities are greatly suppressed. Combined with the system's dissipation, the resonator can emit its energy in antibunched bundles of $N$ strongly correlated photons with high purity and an in situ tunable emission rate. Such a nonclassical source could be useful in applications in the field of quantum information science.
\end{abstract}

DOI: 10.1103/PhysRevResearch.3.043020

\section{INTRODUCTION}

In recent years, Josephson photonics of a dc voltagebiased Josephson junction in series with superconducting microwave resonators has received considerable attention as a bright and robust on-chip source of quantum microwaves [1-8]. Through the ac Josephson effect, the inelastic Cooperpair tunneling through the junction can create a coherent flow of microwave photons inside the resonator [9-12]. The nonclassical radiation with high photon flux can be engineered with a specifically tailored electromagnetic environment [13-17], which requires no external microwave control drives. Experimentally, significant progress in this field has been achieved, such as realizations of masers [18,19], two-mode squeezing [20-22], near-quantum-limited amplifiers [23], and antibunched single-photon emissions [24,25].

The voltage-biased Josephson junction acts as a highly nonlinear driving element to the cavity. Provided that the $N$-photon resonance condition is satisfied (where $N$ is an integer), the transfer of one Cooper pair enables the creation of $N$ cavity photons [26-29]. So a crucial question then arises about whether the strong nonlinearity of this light-charge interaction can be exploited for on-demand generation of $N$-photon states, which is a long-standing goal in quantum information science [30-34]. Because the harmonic resonator has an infinite number of evenly spaced quantum levels, the

\footnotetext{
*These authors contributed equally to this work.

$\dagger$ flli@mail.xjtu.edu.cn

Published by the American Physical Society under the terms of the Creative Commons Attribution 4.0 International license. Further distribution of this work must maintain attribution to the author(s) and the published article's title, journal citation, and DOI.
}

nonlinear cavity drive will inevitably cause the transitions to the higher Fock states. Therefore, it is challenging to directly utilize this device to efficiently generate and manipulate the desired $N$-photon state.

In this work, we introduce an extra charge qubit into the circuit of a dc voltage-biased Josephson junction coupled to a microwave resonator and aim to control the emission of the resonator in the form of antibunched $N$-photon bundles. The central idea is to excite the charge qubit and, at the same time, to populate the resonator with $N$ photons for each resonant tunneling Cooper pair. Meanwhile, a charge qubit with strong anharmonicity is utilized to regulate the tunneling behavior of the Cooper pairs; that is, the presence of $N$ photons in the resonator prevents the next tunnel event. In this way, a superRabi oscillation can be realized between the $|0, g\rangle$ and $|N, e\rangle$ states with vastly different energies, where the resonator's transitions to the higher Fock states are greatly suppressed. Combined with the system's dissipation, the resonator can release its energy in groups of $N$ strongly correlated photons, yielding an antibunched $N$-photon quantum light source. Such a highly nonclassical emitter is of fundamental interest and lies at the heart of many quantum technological applications, such as beating the diffraction limit [35], quantum metrology [36,37], and quantum information processing [38,39].

We recall that the idea of $N$-photon bundle emission was first proposed by Sánchez Muñoz and colleagues with a cavity mode coupled to a strongly driven two-level atom [40] and attracted attention in a series of subsequent studies [41-48]. Here our scheme takes advantage of the nonlinear dynamics of the inelastic Cooper-pair tunneling, where the Josephson junction as a voltage-to-frequency converter is explored to realize the $N$-photon bundle emission with high purity and an in situ tunable rate. It thus differs fundamentally from the previous proposals [40-48]. The present work offers a strategy for the generation of nonclassical microwave radiations with Josephson photonics circuits. 


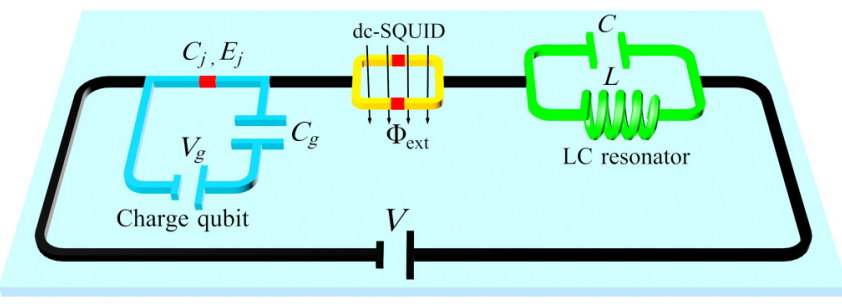

FIG. 1. Schematic of the proposed setup. A dc-SQUID composed of two identical Josephson junctions is coupled to both an $L C$ resonator and a charge qubit via the biased voltage $V$.

\section{MODEL}

As illustrated in Fig. 1, the proposed model consists of a dc voltage-biased dc superconducting quantum interference device (dc-SQUID) in series with an $L C$ resonator and a charge qubit. We consider the situation in which the biased voltage $V$ is smaller than the gap voltage $2 \Delta_{s} / e\left(\Delta_{S}\right.$ is the superconducting gap), where no quasiparticle excitation can be produced in the superconducting electrodes. The charge qubit is a Cooper-pair box that has a tunnel junction with capacitance $C_{j}$ and Josephson coupling energy $E_{j}$; that is, a gate voltage $V_{g}$ as a control parameter is coupled to the system via a gate capacitor $C_{g}$ [49-52]. The dc-SQUID is treated as a tunable Josephson junction with the effective Josephson energy $E_{\mathrm{J}}=2 E_{J 0} \cos \left(\pi \Phi_{\text {ext }} / \Phi_{0}\right)$ [22], which can be varied by controlling the magnetic flux $\Phi_{\text {ext }}$ penetrating the loop ( $\Phi_{0}=h / 2 e$ is the magnetic flux quantum). The superconducting cavity could be realized either as a lumped element oscillator [24] or as the fundamental mode of a transmission line resonator [25]. Since the voltage $V$ locks the total phase of the three subunits, the charge qubit and resonator are coupled to each other via the phase difference across the dc-SQUID.

If the charge qubit is operated at an optimal working point $C_{g} V_{g} / 2 e=1 / 2$, the Hamiltonian of the whole circuit is given by (see Appendix A; hereafter $\hbar=1$ )

$$
H=\frac{1}{2} \delta \sigma_{z}+\omega a^{\dagger} a-E_{J} \cos \left[\omega_{J} t+\eta_{1}+2 \lambda\left(a^{\dagger}+a\right)\right],
$$

where the charge qubit with strong anharmonicity has been reduced to a two-level system of an excited state $|e\rangle$ and a ground state $|g\rangle$; that is, $\sigma_{z}=|e\rangle\langle e|-| g\rangle\langle g|$ is the Pauli matrix, and $\delta$ is the energy splitting. $a^{\dagger}(a)$ is the creation (annihilation) operator of the photons, and $\omega=1 / \sqrt{L C}$ denotes the eigenfrequency of the resonator. The third interaction term couples the Cooper-pair transfer to the excitation of the qubit and resonator. $\omega_{J}=2 \mathrm{eV} / \hbar$ is the Josephson frequency, and $\eta_{1}$ is the phase difference across the qubit's junction. The parameter $\lambda=\sqrt{\pi Z / R_{K}}$ sets the magnitude of zero-point displacement of the resonator, where $Z=\sqrt{L / C}$ is the characteristic impedance, and $R_{K}=h / e^{2}$ is the resistance quantum.

\section{SUPER-RABI OSCILLATION}

Using the Hamiltonian (1), we now derive the effective $N$-photon coupling Hamiltonian that can generate super-Rabi oscillations between the states with an $N$ difference in their photon number. In the interaction picture, we have (see (a)

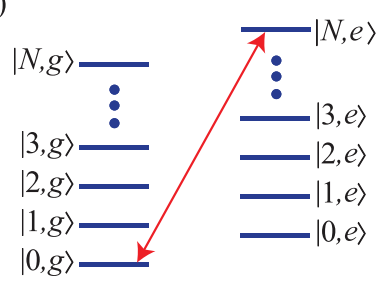

Bare energy structure

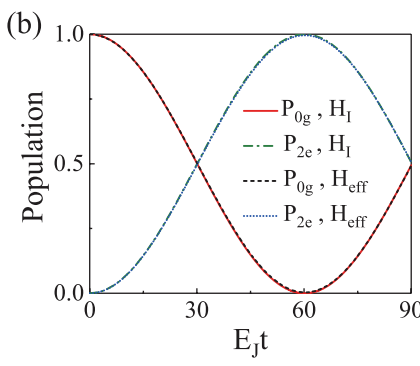

FIG. 2. (a) Bare energy structure of the qubit and resonator, where the red arrow indicates a super-Rabi oscillation between $|0, g\rangle$ and $|N, e\rangle$ states. (b) Dynamics of the state populations $P_{0 g}$ and $P_{2 e}$ for the two-photon resonance; that is, the red solid and green dash-dotted curves are simulated with the original Hamiltonian $H_{\mathrm{I}}$, while the black dashed and blue dotted curves are achieved with the effective Hamiltonian $H_{\text {eff }}$. The parameters are chosen to be $\omega / 2 \pi=7 \mathrm{GHz}$, $\delta / 2 \pi=5 \mathrm{GHz}, E_{J} / 2 \pi=0.7 \mathrm{GHz}$, and $\lambda=0.2$.

Appendix B)

$$
H_{\mathrm{I}}=-\frac{E_{\mathrm{J}}}{4} e^{-i \omega_{\mathrm{J}} t}\left(\sigma^{-} e^{-i \delta t}-\sigma^{+} e^{i \delta t}-\sigma_{z}\right) D[\alpha(t)]+\text { H.c. },
$$

where $\sigma^{+}=|e\rangle\langle g|\left(\sigma^{-}=|g\rangle\langle e|\right)$ is the spin-ladder operator and $D[\alpha(t)]=\exp \left[\alpha(t) a^{\dagger}-\alpha^{*}(t) a\right]$ is the cavity displacement operator with time-dependent amplitude $\alpha(t)=$ $-2 i \lambda e^{i \omega t}$. The Hamiltonian $H_{\mathrm{I}}$ describes a highly nonlinear drive, which is akin to the multiphoton process in trapped ions [53]. By choosing an appropriate voltage $V$, we can set the $N$-photon resonance condition $\omega_{J}=\delta+N \omega$. In the rotating-wave approximation, we obtain the effective Hamiltonian $H_{\mathrm{eff}}=\sum_{m=0}^{\infty} H_{m}$ under the Fock basis (see Appendix B), where

$$
H_{m}=g_{m}^{m+N}|m+N, e\rangle\langle m, g|+\text { H.c. }
$$

and $g_{m}^{m+N}=E_{J} \beta_{m}^{m+N} / 4$ is the $N$-photon resonance Rabi frequency with

$$
\beta_{m}^{m+N}=\sqrt{\frac{m !}{(m+N) !}}(-2 i \lambda)^{N} e^{-2 \lambda^{2}} L_{m}^{(N)}\left(4 \lambda^{2}\right) .
$$

In Eq. (4), $L_{m}^{(N)}\left(4 \lambda^{2}\right)$ is a Laguerre polynomial.

In the absence of dissipation, each $H_{m}$ in $H_{\text {eff }}$ can induce a coherent $N$-photon transition from the state $|m, g\rangle$ to $|m+N, e\rangle$. So the Cooper pair can tunnel to excite the qubit and the resonator with $N$ photons simultaneously. Note that, if the system is initially prepared in the ground state $|0, g\rangle$, the Hamiltonian $H_{0}$ will govern the evolution of the wave function, which is restricted to a truncated Hilbert subspace of a super-Rabi oscillation $|0, g\rangle \longleftrightarrow|N, e\rangle$, as depicted in Fig. 2(a). Hence, the charge qubit under the two-level approximation is crucial for preventing the excitations of the cavity to the higher Fock states. Similar to the mechanism of multiphoton blockade [54-58], the absorption of $N$ cavity photons will impede the further tunneling of the Cooper pair and inhibit the absorption of the next $N$ photons. Thus, the cavity contains only $N$ photons or none, and all other possibilities are strongly suppressed. This is the essential point for 
implementing a quantum microwave source that emits energy strictly by $N$-photon quanta.

To exhibit the multiphoton Rabi oscillation, we numerically solve the Schrödinger equation for the two-photon resonance, where the initial state is $|0, g\rangle$. The time evolution of the populations $P_{0 g}$ in the state $|0, g\rangle$ and $P_{2 e}$ in the state $|2, e\rangle$ is presented in Fig. 2(b). Strikingly, perfect super-Rabi oscillation $|0, g\rangle \longleftrightarrow|2, e\rangle$ is observed with both the original Hamiltonian $H_{\mathrm{I}}$ and the effective Hamiltonian $H_{\text {eff }}$. Moreover, we just need to adjust the biased voltage $V$ to match the different $N$-photon resonance conditions.

\section{ANTIBUNCHED $N$-PHOTON BUNDLE EMISSION}

To trigger the $N$-photon bundle emission, we have to consider the dissipation of the system, which will transform the $N$-photon state into $N$ strongly correlated photons outside of the cavity. In the Born-Markov approximation, the dynamics of the system is determined by the master equation

$$
\frac{d \rho}{d t}=-i\left[H_{\mathrm{I}}, \rho\right]+\frac{\kappa}{2} \mathcal{L}[a] \rho+\frac{\gamma}{2} \mathcal{L}[\sigma] \rho,
$$

where $\mathcal{L}[o] \rho=2 o \rho o^{\dagger}-o^{\dagger} o \rho-\rho o^{\dagger} o$ is the Lindblad operator for a given operator $o$ and $\kappa(\gamma)$ denotes the energy damping rate of the cavity (qubit). We have assumed that the cavity and qubit are coupled to the environment independently in the weak-coupling regime, i.e., $\omega, \delta \gg \gamma, \kappa$. Additionally, we have neglected the thermal excitation, which is about $\sim 10^{-7}$ at the working temperature of $20 \mathrm{mK}$.

The underlying principle of the $N$-photon source is that a former Cooper-pair tunneling event blocks the latter one, which gives rise to an antibunched $N$-photon bundle emission. More specifically, the tunneling of a single Cooper pair extracts energy quanta from the voltage and constructs a coherent super-Rabi oscillation; that is, the system will evolve to a superposition state of $|0, g\rangle$ and $|N, e\rangle$. In the presence of dissipation, each photon in $|N, e\rangle$ has a chance to leave the cavity, which is a probabilistic event determined by Rabi frequency $g_{0}^{N}$ and photon loss rate $\kappa$. Once one photon leaks out of the cavity, the system collapses to the state $|N-1, e\rangle$, which will disrupt the giant Rabi oscillation due to its insufficient energy. Consequently, the remaining photons have no choice but to leave the cavity in rapid succession. As a result, a bundle of $N$ cascaded itinerant photons is emitted, but with an intrinsic temporal structure; that is, the first photon emitted with the time $1 / N \kappa$ is closely followed by the subsequent one, $1 /(N-1) \kappa$, until the last one, $1 / \kappa$. The total emission time of the bundle is $\tau_{B}=\sum_{i=1}^{N} 1 / i \kappa$ (smaller than $3 / \kappa$ for $N=10$ ). After all photons have left out of the cavity, the system is in the state $|0, e\rangle$. Only after direct emission of the qubit within its coherence time $\tau_{Q}=1 / \gamma$ can the second Cooper pair traverse to reconstruct the Rabi oscillation for the next emission of a bundle.

The above physical picture is valid only when the condition $\kappa \gg \gamma$ is satisfied. First, this is because the state $|N, e\rangle$ has another dissipative channel via the charge qubit's spontaneous emission. If $\kappa$ is not much larger than $\gamma$, there will be a non-negligible probability for $|N, e\rangle$ to dissipate into the state $|N, g\rangle$. Then, the Rabi dynamics controlled by the subunit $H_{N}$ in Eq. (3) will further shuffle population from $|N, g\rangle$ to (a)

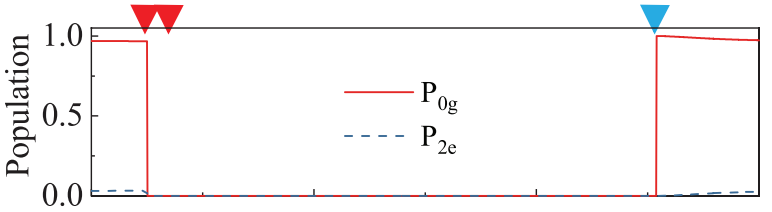

(b)

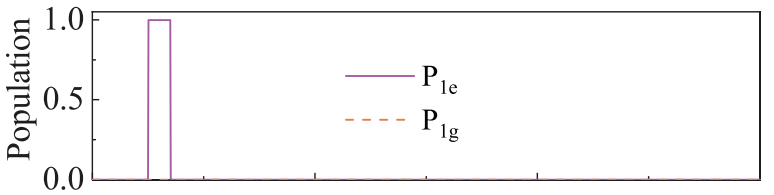

(c)

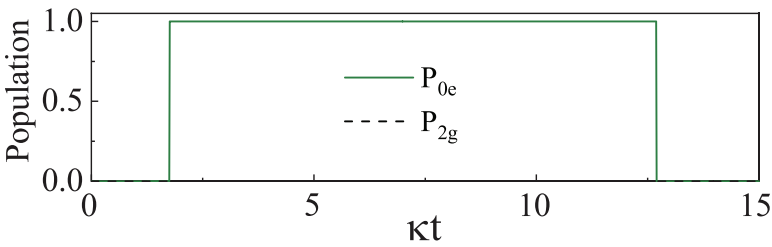

FIG. 3. A tiny fraction of one quantum trajectory showing a complete period of two-photon emission, where the red triangles denote the successive one-photon emissions and the blue one indicates the flip of the qubit. The damping rates are $\kappa / 2 \pi=0.1 \mathrm{GHz}$ and $\gamma=\kappa / 20$. The other parameters are chosen to be the same as those in Fig. 2.

the state $|2 N, e\rangle$, contaminating the pure $N$-photon emission. More importantly, the timescales of $\tau_{Q} \gg \tau_{B}$ guarantee the emitted bundles are well separated, which leads to the desired antibunching. On the other hand, we cannot make an arbitrarily small $\gamma$. At the extreme case of $\gamma \rightarrow 0$, the system is close to being a completely antibunched $N$-photon bundle emitter, but with an extremely low emission rate because of the infinite reloading time. Therefore, $\gamma$ is a key factor for determining the quantum character of the emission. We have to balance $\kappa$ and $\gamma$ to ensure an $N$-photon source with a purity and emission rate that are both relatively high concurrently.

To well understand the photon radiating process, we now turn to the Monte Carlo simulations with $\gamma=\kappa / 20$ and track individual trajectories of the system. The emission event is recorded whenever the system undergoes a quantum jump. As shown in Figs. 3(a)-3(c), we display a tiny fraction of one quantum trajectory for the two-photon resonance. At first, the super-Rabi oscillation causes the system to be in the superposition state of $|0, g\rangle$ and $|2, e\rangle$. As time goes on, the cavity emits one photon, denoted by the first red triangle in Fig. 3(a), resulting in the collapse of the wave function into the state $|1, e\rangle$ with almost unit probability, as seen in Fig. 3(b). Immediately, the second photon is emitted within the cavity lifetime (the second red triangle). Thus, a bundle of two photons is emitted in a very short temporal window. As expected in Fig. 3(c), the system then stays in the state $|0, e\rangle$ for a long time until the flip of the qubit (the blue triangle), after which it will return to the ground state $|0, g\rangle$ and start a new period for emitting the next photon pair.

It is now clear that the $N$-photon bundle stems from the radiative cascade of a Fock state $|N\rangle$. So the emitted $N$ photons in a bundle are strongly correlated. In Fig. 4(a), the zerodelay photon correlation functions $g^{(n)}=\left\langle a^{\dagger n} a^{n}\right\rangle /\left\langle a^{\dagger} a\right\rangle^{n}$ are plotted, indicating strong correlations of the radiated photons. Remarkably, at each $N$-photon resonance point $\Delta=N \omega$, a 

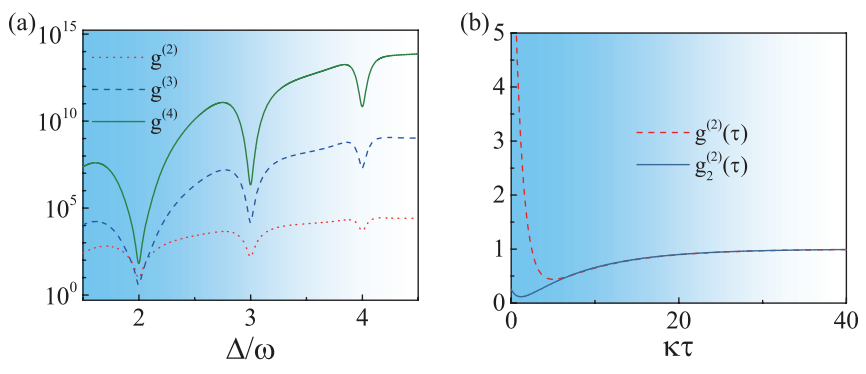

FIG. 4. (a) Zero-delay $n$ th-order photon correlation functions $g^{(n)}$ versus $\Delta / \omega$, where $\Delta=\omega_{J}-\delta$. (b) The standard second-order correlation function $g^{(2)}(\tau)$ and the generalized second-order correlation function $g_{2}^{(2)}(\tau)$ versus $\kappa \tau$ for the two-photon resonance. The parameters are chosen to be the same as those in Fig. 3.

sharp dip instead of a bunching peak is observed for each $g^{(n)}$, which is solid evidence that the cavity emits its energy in antibunched $N$-photon bundles. To reveal the quantum statistics of the bundles, we adopt the generalized second-order correlation function [40]

$$
g_{N}^{(2)}(\tau)=\frac{\left\langle a^{\dagger N}(0) a^{\dagger N}(\tau) a^{N}(\tau) a^{N}(0)\right\rangle}{\left\langle\left(a^{\dagger N} a^{N}\right)(0)\right\rangle\left\langle\left(a^{\dagger N} a^{N}\right)(\tau)\right\rangle}
$$

to characterize the bunching or antibunching, where the $N$ photon events are considered the basic unit of the emission. As simulated in Fig. 4(b), the numerical result for $g_{2}^{(2)}(\tau)$ shows that the system behaves as an antibunched photon-pair emitter for $\gamma=\kappa / 20$, while the standard $g^{(2)}(\tau)$ fails to capture the correlation between the separated photon pairs.

\section{PURITY AND EMISSION RATE}

An ideal $N$-photon emitter emits only bundles of $N$ photons. In a realistic circumstance, however, it may experience undesirable processes, such as single-photon emission or excitation to the $|2 N\rangle$ Fock state, which will produce bundles with other photon numbers. To quantify the amount of $N$-photon emission, we introduce the purity and the emission rate [44], which are the most important features of such an emitter. The purity is defined as

$$
\pi_{N}=\frac{n_{a}^{(I, N)}}{\sum_{i=1}^{N} n_{a}^{(i)}}
$$

where $n_{a}^{(I, N)}$ is the cavity population of the ideal $N$-photon emission and $n_{a}^{(i)}$ is the mean photon number accumulated by all the possible $i$-photon transitions. Obviously, $\pi_{N}$ represents the percentage of the emission to be $N$-photon bundles. The emission rate is given by $S_{N}=\kappa n_{a}^{(I, N)}$.

The purity $\pi_{2}$ and rate $S_{2}$ of the two-photon emission versus $\Delta V$ and $\gamma$ are respectively shown in Figs. 5(a) and 5(b), where $\Delta V$ is the voltage that deviated from the resonance one. We can see that, in the range $\gamma / \kappa \in[0,0.1], \pi_{2}$ slowly decreases with the increase of $\gamma$, while $S_{2}$ has the opposite behavior. As stated before, there is, indeed, a trade-off between the purity and the emission rate. Experimentally, when an external control line is coupled to the charge qubit, the decay rate $\gamma$ can be adjusted by tuning the coupling strength between them $[59,60]$. This allows us to have an in situ tunable emis-
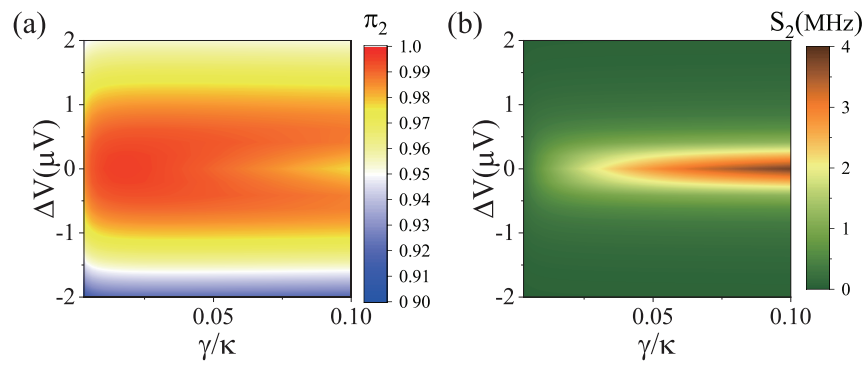

FIG. 5. Purity and rate of the two-photon emission. (a) $\pi_{2}$ and (b) $S_{2}$ versus the imperfect tuning $\Delta V$ and the qubit's decay rate $\gamma$ for a fixed cavity decay rate $\kappa / 2 \pi=0.1 \mathrm{GHz}$. The other parameters are chosen to be the same as those in Fig. 2.

sion rate. With the currently available parameters $\omega / 2 \pi=$ $7 \mathrm{GHz}, \delta / 2 \pi=5 \mathrm{GHz}, E_{J} / 2 \pi=0.7 \mathrm{GHz}, \kappa / 2 \pi=0.1 \mathrm{GHz}$, $\gamma / 2 \pi=0.005 \mathrm{GHz}$, and $\lambda=0.2$, two-photon emission with a purity $\pi_{2} \sim 99 \%$ and a rate $S_{2} \sim 2.78 \mathrm{MHz}$ can be achieved. Note that, as the number $N$ gets larger, the $N$-photon coupling rate is considerably reduced (see Appendix C). So the other off-resonance $i$-photon process $(i \neq N)$ will gradually dominate the dynamics and spoil the $N$-photon character of the output field. Nevertheless, we can still reach about $99 \%$ of the three-photon emission with a rate $\sim 0.22 \mathrm{MHz}$ (see Appendix C).

Finally, we perform Monte Carlo simulations over 40 quantum trajectories to record the photon clicks, and there are enough data to simulate a real situation with radiated photons. In Fig. 6, a series of photon emission events for the twophoton resonance is illustrated. Apart from two single-photon emissions and one four-photon emission, all the rest of the 225 events are two-photon emissions. According to these data, we can readily get an efficiency $\sim 99 \%$ for the two-photon emission with a rate $\sim 2.81 \times 10^{6}$ counts/s, which is pretty consistent with the numerical results for $\pi_{2}$ and $S_{2}$.

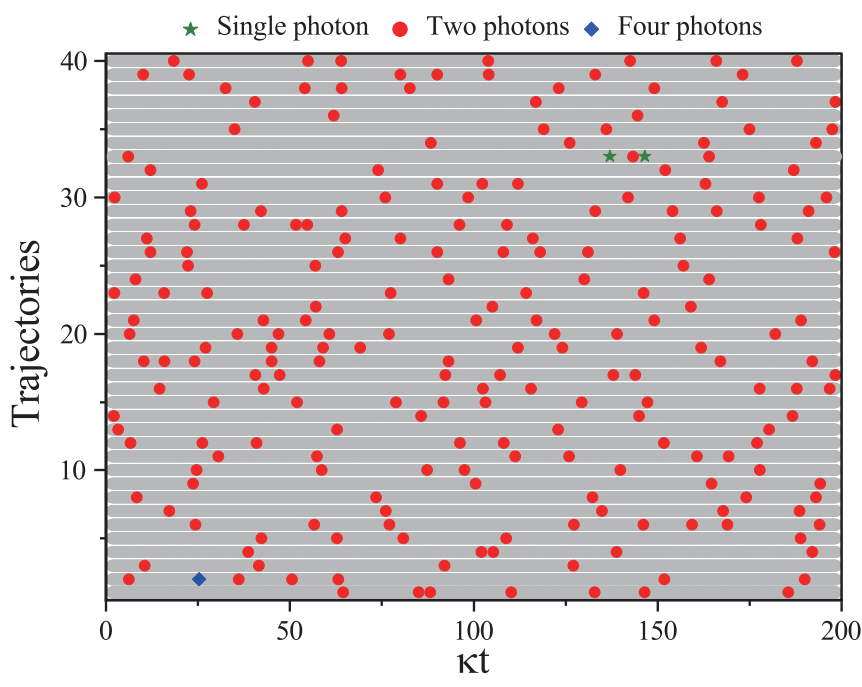

FIG. 6. A series of emission events extracted from the Monte Carlo simulations over 40 quantum trajectories for the two-photon resonance. The single-, two-, and four-photon emissions are denoted by green stars, red circles, and the blue diamond, respectively. The parameters are chosen to be the same as those in Fig. 3 . 


\section{CONCLUSIONS}

We have proposed an efficient method for the generation of antibunched $N$-photon bundles in a Josephson photonics device. By embedding a charge qubit in the circuit of a dc voltage-biased dc-SQUID coupled to a superconducting cavity, we can establish a super-Rabi oscillation between the $|0, g\rangle$ and $|N, e\rangle$ states through Cooper-pair tunneling, where the two-state charge qubit plays a key role in inhibiting the excitation of the cavity to higher Fock states. Together with the system's dissipation, the cavity can continuously radiate antibunched bundles of $N$ strongly correlated photons; that is, almost pure two- and three-photon emissions with the rates on the order of megahertz can be reached with the current technology. This nonclassical source could be instrumental for various quantum technological applications.

\section{ACKNOWLEDGMENTS}

This work was supported by the National Nature Science Foundation of China (Grants No. 11704306 and No. 12074307). Part of the numerical simulations was performed with the open-source PYTHON package [61].

\section{APPENDIX A: DETAILED DERIVATION OF EQ. (1)}

As shown in Fig. 1, the Hamiltonian of the system follows from the three subunits

$$
\begin{aligned}
\tilde{H}= & 4 E_{c}\left(n_{1}-n_{g}\right)^{2}-E_{j} \cos \eta_{1} \\
& +\frac{q^{2}}{2 C}+\left(\frac{\hbar}{2 e}\right)^{2} \frac{\phi^{2}}{2 L} \\
& -E_{J} \cos \eta_{2}-2 e\left(V-V_{\mathrm{res}}-V_{q}\right) n_{2} .
\end{aligned}
$$

The first two terms describe the charge qubit part with the dimensionless gate charge $n_{g}=C_{g} V_{g} / 2 e$ as a control parameter. $E_{c}=e^{2} / 2\left(C_{j}+C_{g}\right)$ is the single-electron charging energy, and $E_{j}$ is the Josephson coupling energy. $n_{1}$ is the number of Cooper pairs, and $\eta_{1}$ is the phase difference across the junction. The second two terms are the resonator part, where $q$ and $\phi$ denote the charge and phase operators acting on the capacitance $C$ and inductance $L$, respectively. The last two terms represent the dc-SQUID part, where $E_{J}$ is the effective Josephson coupling energy, $\eta_{2}$ is the phase difference, and $n_{2}$ counts the number of transferred Cooper pairs [4,5]. These three sets of conjugate variables obey $\left[\eta_{1}, n_{1}\right]=i$, $[\phi, q]=2 i e$, and $\left[\eta_{2}, n_{2}\right]=i$. According to Kirchhoff's rules, the voltage across the dc-SQUID is $V-V_{\text {res }}-V_{q}$, where $V_{\text {res }}=-\hbar \dot{\phi} / 2 e$ and $V_{q}=-\hbar \dot{\eta}_{1} / 2 e$ are the voltage drops at the resonator and qubit, respectively. By utilizing the classical phase-voltage relation $\dot{\eta}_{2}=V-V_{\text {res }}-V_{q}$, we can rewrite the dc-SQUID's Josephson coupling term as $-E_{J} \cos \left(\omega_{J} t+\eta_{1}+\right.$ $\phi)[4,5]$, where $\omega_{J}=2 \mathrm{eV} / \hbar$ is the Josephson frequency. Since the voltage $V$ locks the total phase of the three subunits, the nonlinear coupling between the charge qubit and resonator is induced via the phase difference across the dc-SQUID.

To obtain the desired coupling, we perform a timedependent gauge transformation $U(t)=\exp \left[i\left(\omega_{J} t+\eta_{1}+\right.\right.$ $\phi) n_{2}$ ] to Eq. (A1). By means of the formula $H=$
$U(t)^{\dagger} \tilde{H} U(t)+i \hbar \frac{d U(t)^{\dagger}}{d t} U(t)$, we can get the Hamiltonian

$$
\begin{aligned}
H= & 4 E_{C}\left(\tilde{n}_{1}-n_{g}\right)^{2}-E_{j} \cos \eta_{1}+\frac{\tilde{q}^{2}}{2 C}+\left(\frac{\hbar}{2 e}\right)^{2} \frac{\phi^{2}}{2 L} \\
& -E_{J} \cos \left(\omega_{J} t+\eta_{1}+\phi\right) .
\end{aligned}
$$

In the equation above, $\tilde{n}_{1}=n_{1}+n_{2}\left(\tilde{q}=q+2 n_{2} e\right)$ is the new number (charge) operator, which is caused by the charge fluctuations relative to the flow of Cooper pairs through the dc-SQUID. Even so, the transformed operator $\tilde{n}_{1}(\tilde{q})$ is still conjugate to $\eta_{1}(\phi)$.

In the charge regime $E_{c} \gg E_{j}$, the Hamiltonian describing the charge qubit takes the form [49-52]

$$
\begin{aligned}
H_{q}= & \sum_{n}\left[4 E_{C}\left(\tilde{n}_{1}-n_{g}\right)|n\rangle\langle n|\right. \\
& \left.-\frac{E_{j}}{2}(|n+1\rangle\langle n|+| n\rangle\langle n+1|)\right],
\end{aligned}
$$

where $|n\rangle$ is the eigenstate of the number operator $\tilde{n}_{1}$. At the degeneracy point $n_{g}=1 / 2$, only the two lowest-energy charge states of $|0\rangle$ and $|1\rangle$ are relevant, while all other charge states with a much higher energy can be ignored. Then, the charge qubit is reduced as a two-state system with $H_{q}=$ $\frac{1}{2} \hbar \delta \sigma_{z}$, where $\delta=E_{j} / \hbar$ is the transition frequency and $\sigma_{z}=$ $|e\rangle\langle e|-| g\rangle\langle g|$ is the Pauli operator in the basis of excited state $|e\rangle=(|1\rangle-|0\rangle) / \sqrt{2}$ and ground state $|g\rangle=(|1\rangle+|0\rangle) / \sqrt{2}$.

Furthermore, we introduce the annihilation $a$ and creation $a^{\dagger}$ operators

$$
a=\sqrt{\frac{\hbar}{8 Z e^{2}}}\left(\phi+\frac{i 2 e Z \tilde{q}}{\hbar}\right), \quad a^{\dagger}=\sqrt{\frac{\hbar}{8 Z e^{2}}}\left(\phi-\frac{i 2 e Z \tilde{q}}{\hbar}\right),
$$

where $Z=\sqrt{L / C}$ is the characteristic impedance. In terms of $a$ and $a^{\dagger}$, the Hamiltonian of the $L C$ resonator can be quantized as $H_{r}=\hbar \omega a^{\dagger} a$, where $\omega=\sqrt{1 / L C}$ is the resonance frequency.

By substituting $H_{q}, H_{r}$, and $\phi=2 \lambda\left(a^{\dagger}+a\right)$ into Eq. (A2), we obtain (hereafter $\hbar=1$ )

$$
H=\frac{1}{2} \delta \sigma_{z}+\omega a^{\dagger} a-E_{J} \cos \left[\omega_{J} t+\eta_{1}+2 \lambda\left(a^{\dagger}+a\right)\right] .
$$

This is just Eq. (1) in the main text. Here the parameter $\lambda=$ $\sqrt{\pi Z / R_{K}}$ sets the magnitude of the zero-point displacement of the resonator ( $R_{K}=h / e^{2}$ is the resistance quantum).

\section{APPENDIX B: DETAILED DERIVATION OF THE $N$-PHOTON COUPLING HAMILTONIAN}

We now give more details on deriving the $N$-photon coupling Hamiltonian. In the interaction picture with respect to the frame rotating $\exp \left[-i t\left(\frac{1}{2} \delta \sigma_{z}+\omega a^{\dagger} a\right)\right]$, the Hamiltonian in Eq. (A5) will yield

$$
H_{\mathrm{I}}=-\frac{E_{\mathrm{J}}}{4} e^{-i \omega_{\mathrm{J}} t}\left(\sigma^{-} e^{-i \delta t}-\sigma^{+} e^{i \delta t}-\sigma_{z}\right) D[\alpha(t)]+\text { H.c. },
$$

where we have used the relation $e^{-i \eta_{1}}=\left(\sigma^{-}-\sigma^{+}-\sigma^{z}\right) / 2$ and $\sigma^{+}=|e\rangle\langle g|\left(\sigma^{-}=|g\rangle\langle e|\right)$ is the spin-ladder operator. In 


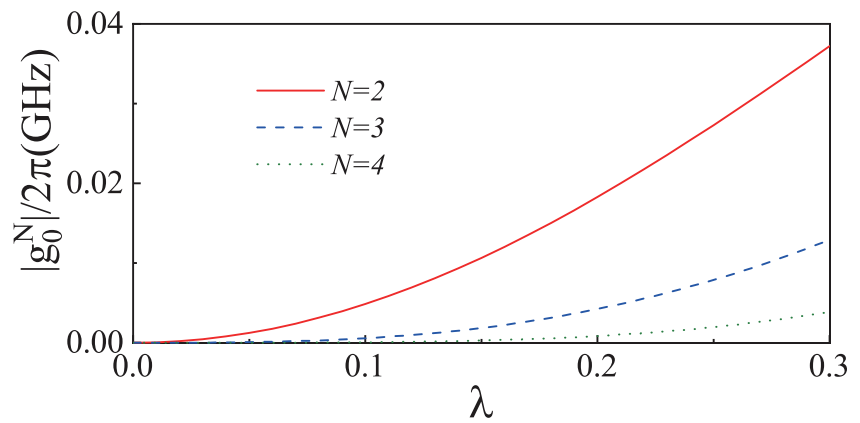

FIG. 7. The different $N$-photon coupling rates $\left|g_{0}^{N}\right|$ versus the cavity's zero-point fluctuation $\lambda$, where we choose the parameter $E_{J} / 2 \pi=0.7 \mathrm{GHz}$.

addition, $D[\alpha(t)]=\exp \left[\alpha(t) a^{\dagger}-\alpha^{*}(t) a\right]$ is the cavity displacement operator with time-dependent amplitude $\alpha(t)=$ $-2 i \lambda e^{i \omega t}$, which can be directly expanded in the Fock basis [62]

$$
\begin{aligned}
D[\alpha(t)]= & \sum_{m=0}^{\infty}\left(\sum_{l=0}^{\infty} \beta_{m}^{m+l}|m+l\rangle\langle m| e^{i l \omega t}\right. \\
& \left.+\sum_{l^{\prime}=1}^{\infty} \beta_{m}^{m+l^{\prime}}|m\rangle\left\langle m+l^{\prime}\right| e^{-i l^{\prime} \omega t}\right),
\end{aligned}
$$

with

$$
\beta_{m}^{m+l}=\sqrt{\frac{m !}{(m+l) !}}(-2 i \lambda)^{l} e^{-2 \lambda^{2}} L_{m}^{(l)}\left(4 \lambda^{2}\right) .
$$

In Eq. (B3), $L_{m}^{(l)}\left(4 \lambda^{2}\right)$ is a Laguerre polynomial. By choosing an appropriate voltage $V$, we can set the $N$-photon resonance condition $\omega_{J}=\delta+N \omega$. For the parameters $\omega_{\mathrm{J}}, \delta, \omega \gg$ $E_{J} \beta_{m}^{m+l} / 4$, we can make a rotating-wave approximation to discard those rapidly oscillating terms and derive the effective Hamiltonian

$$
H_{\text {eff }}=\sum_{m=0}^{\infty} H_{m}=\sum_{m=0}^{\infty} g_{m}^{m+N}|m+N, e\rangle\langle m, g|+\text { H.c. },
$$

where $g_{m}^{m+N}=E_{J} \beta_{m}^{m+N} / 4$ is the $N$-photon coupling rate that enables a direct transition from $|m, g\rangle$ to the state $|m+N, e\rangle$.
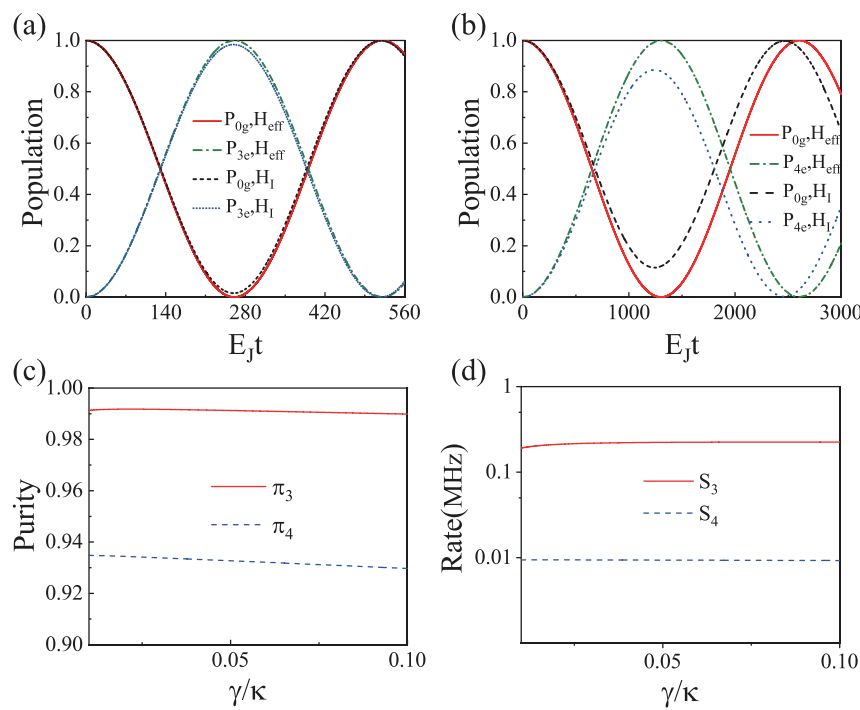

FIG. 8. (a) and (b) Dynamics of the state populations $P_{0 g}$ and $P_{N e}$ for the three- and four-photon resonances without regard to dissipation; that is, the red solid and green dash-dotted curves are simulated with the effective Hamiltonian $H_{\text {eff }}$, while the black dashed and blue dotted curves are achieved with the original Hamiltonian $H_{\mathrm{I}}$. (c) and (d) Purities and rates of the three- and four-photon emissions. The parameters are chosen to be the same as those in Fig. 5.

\section{APPENDIX C: THE PURITIES AND RATES OF THE THREE- AND FOUR-PHOTON EMISSIONS}

In the main text, a thorough study of the two-photon emission was made. Here we investigate the purities and rates of the three- and four-photon emissions. As shown in Fig. 7, with the increase of $N$, the $N$-photon coupling rate $\left|g_{0}^{N}\right|$ is considerably reduced in the range $\lambda \in[0,0.3]$. So the other off-resonance $i$-photon process $(i \neq N)$ will gradually dominate the dynamics and spoil the pure $N$-photon bundle emission. The super-Rabi oscillations $|0, g\rangle \longleftrightarrow|N, e\rangle$ for the three- and four-photon resonance are shown in Figs. 8(a) and $8(b)$, respectively. It is seen that the rotating-wave approximation does not work well for the case $N=4$, where the other off-resonance processes play a non-negligible role. Figures 8(c) and 8(d) display the purities and rates of the three- and four-photon emissions. As the number $N$ gets larger, both the purity and emission rate are obviously reduced. Nevertheless, we can still reach about $99 \%$ of the three-photon emission with a rate $\sim 0.22 \mathrm{MHz}$.
[1] M. Hofheinz, F. Portier, Q. Baudouin, P. Joyez, D. Vion, P. Bertet, P. Roche, and D. Esteve, Bright Side of the Coulomb Blockade, Phys. Rev. Lett. 106, 217005 (2011).

[2] C. Padurariu, F. Hassler, and Y. V. Nazarov, Statistics of radiation at Josephson parametric resonance, Phys. Rev. B 86, 054514 (2012).

[3] A. D. Armour, M. P. Blencowe, E. Brahimi, and A. J. Rimberg, Universal Quantum Fluctuations of a Cavity Mode Driven by a Josephson Junction, Phys. Rev. Lett. 111, 247001 (2013).
[4] V. Gramich, B. Kubala, S. Rohrer, and J. Ankerhold, From Coulomb-Blockade to Nonlinear Quantum Dynamics in a Superconducting Circuit with a Resonator, Phys. Rev. Lett. 111, 247002 (2013).

[5] M. Trif and P. Simon, Photon cross-correlations emitted by a Josephson junction in two microwave cavities, Phys. Rev. B 92, 014503 (2015).

[6] M. Koppenhöfer, J. Leppäkangas, and M. Marthaler, Creating photon-number squeezed strong microwave fields by a Cooperpair injection laser, Phys. Rev. B 95, 134515 (2017). 
[7] S. H. Simon and N. R. Cooper, Theory of the Josephson Junction Laser, Phys. Rev. Lett. 121, 027004 (2018).

[8] W. T. Morley, A. Di Marco, M. Mantovani, P. Stadler, W. Belzig, G. Rastelli, and A. D. Armour, Theory of double Cooper-pair tunneling and light emission mediated by a resonator, Phys. Rev. B 100, 054515 (2019).

[9] S. Meister, M. Mecklenburg, V. Gramich, J. T. Stockburger, J. Ankerhold, and B. Kubala, Resonators coupled to voltagebiased Josephson junctions: From linear response to strongly driven nonlinear oscillations, Phys. Rev. B 92, 174532 (2015).

[10] A. D. Armour, B. Kubala, and J. Ankerhold, Noise switching at a dynamical critical point in a cavity-conductor hybrid, Phys. Rev. B 96, 214509 (2017).

[11] H. Wang, M. P. Blencowe, A. D. Armour, and A. J. Rimberg, Quantum dynamics of a Josephson junction driven cavity mode system in the presence of voltage bias noise, Phys. Rev. B 96, 104503 (2017).

[12] B. Kubala, J. Ankerhold, and A. D Armour, Electronic and photonic counting statistics as probes of non-equilibrium quantum dynamics, New J. Phys. 22, 023010 (2020).

[13] J. Leppäkangas, G. Johansson, M. Marthaler, and M. Fogelström, Nonclassical Photon Pair Production in a VoltageBiased Josephson Junction, Phys. Rev. Lett. 110, 267004 (2013).

[14] J. Leppäkangas, M. Fogelström, A. Grimm, M. Hofheinz, M. Marthaler, and G. Johansson, Antibunched Photons from Inelastic Cooper-Pair Tunneling, Phys. Rev. Lett. 115, 027004 (2015).

[15] A. D. Armour, B. Kubala, and J. Ankerhold, Josephson photonics with a two-mode superconducting circuit, Phys. Rev. B 91, 184508 (2015).

[16] S. Dambach, B. Kubala, V. Gramich, and J. Ankerhold, Timeresolved statistics of nonclassical light in Josephson photonics, Phys. Rev. B 92, 054508 (2015).

[17] J.-R. Souquet and A. A. Clerk, Fock-state stabilization and emission in superconducting circuits using dc-biased Josephson junctions, Phys. Rev. A 93, 060301(R) (2016).

[18] F. Chen, J. Li, A. D. Armour, E. Brahimi, J. Stettenheim, A. J. Sirois, R. W. Simmonds, M. P. Blencowe, and A. J. Rimberg, Realization of a single-Cooper-pair Josephson laser, Phys. Rev. B 90, 020506(R) (2014).

[19] M. C. Cassidy, A. Bruno, S. Rubbert, M. Irfan, J. Kammhuber, R. N. Schouten, A. R. Akhmerov, and L. P. Kouwenhoven, Demonstration of an ac Josephson junction laser, Science 355, 939 (2017)

[20] J.-C. Forgues, C. Lupien, and B. Reulet, Emission of Microwave Photon Pairs by a Tunnel Junction, Phys. Rev. Lett. 113, 043602 (2014).

[21] J.-C. Forgues, C. Lupien, and B. Reulet, Experimental Violation of Bell-Like Inequalities by Electronic Shot Noise, Phys. Rev. Lett. 114, 130403 (2015).

[22] M. Westig, B. Kubala, O. Parlavecchio, Y. Mukharsky, C. Altimiras, P. Joyez, D. Vion, P. Roche, D. Esteve, M. Hofheinz, M. Trif, P. Simon, J. Ankerhold, and F. Portier, Emission of Nonclassical Radiation by Inelastic Cooper Pair Tunneling, Phys. Rev. Lett. 119, 137001 (2017).

[23] S. Jebari, F. Blanchet, A. Grimm, D. Hazra, R. Albert, P. Joyez, D. Vion, D. Estève, F. Portier, and M. Hofheinz, Near-quantumlimited amplification from inelastic Cooper-pair tunnelling, Nat. Electron. 1, 223 (2018).
[24] C. Rolland, A. Peugeot, S. Dambach, M. Westig, B. Kubala, Y. Mukharsky, C. Altimiras, H. le Sueur, P. Joyez, D. Vion, P. Roche, D. Esteve, J. Ankerhold, and F. Portier, Antibunched Photons Emitted by a dc-Biased Josephson Junction, Phys. Rev. Lett. 122, 186804 (2019).

[25] A. Grimm, F. Blanchet, R. Albert, J. Leppäkangas, S. Jebari, D. Hazra, F. Gustavo, J.-L. Thomassin, E. Dupont-Ferrier, F. Portier, and M. Hofheinz, Bright On-demand Source of Antibunched Microwave Photons Based on Inelastic Cooper Pair Tunneling, Phys. Rev. X 9, 021016 (2019).

[26] S. Dambach, B. Kubala, and J. Ankerhold, Generating entangled quantum microwaves in a Josephson-photonics device, New J. Phys. 19, 023027 (2017).

[27] J. Leppäkangas and M. Marthaler, Inelastic scattering of microwave radiation in the dynamical Coulomb blockade, Phys. Rev. B 98, 224511 (2018).

[28] J. Leppäkangas, M. Marthaler, D. Hazra, S. Jebari, R. Albert, F. Blanchet, G. Johansson, and M. Hofheinz, Multiplying and detecting propagating microwave photons using inelastic Cooper-pair tunneling, Phys. Rev. A 97, 013855 (2018).

[29] L. Arndt and F. Hassler, Statistics of radiation due to nondegenerate Josephson parametric down-conversion, Phys. Rev. B 100, 014505 (2019).

[30] M. Hofheinz, E. M. Weig, M. Ansmann, R. C. Bialczak, E. Lucero, M. Neeley, A. D. O'Connell, H. Wang, J. M. Martinis, and A. N. Cleland, Generation of Fock states in a superconducting quantum circuit, Nature (London) 454, 310 (2008).

[31] S. Deléglise, I. Dotsenko, C. Sayrin, J. Bernu, M. Brune, J.-M. Raimond, and S. Haroche, Reconstruction of nonclassical cavity field states with snapshots of their decoherence, Nature (London) 455, 510 (2008).

[32] A. González-Tudela, V. Paulisch, H. J. Kimble, and J. I. Cirac, Efficient Multiphoton Generation in Waveguide Quantum Electrodynamics, Phys. Rev. Lett. 118, 213601 (2017).

[33] S. P. Premaratne, F. C. Wellstood, and B. S. Palmer, Microwave photon Fock state generation by stimulated Raman adiabatic passage, Nat. Commun. 8, 14148 (2017).

[34] W. Wang, L. Hu, Y. Xu, K. Liu, Y. Ma, S.-B. Zheng, R. Vijay, Y. P. Song, L.-M. Duan, and L. Sun, Converting Quasiclassical States Into Arbitrary Fock State Superpositions in a Superconducting Circuit, Phys. Rev. Lett. 118, 223604 (2017).

[35] M. D'Angelo, M. V. Chekhova, and Y. Shih, Two-Photon Diffraction and Quantum Lithography, Phys. Rev. Lett. 87, 013602 (2001).

[36] I. Afek, O. Ambar, and Y. Silberberg, High-NOON states by mixing quantum and classical light, Science 328, 879 (2010).

[37] V. Giovannetti, S. Lloyd, and L. Maccone, Quantum Metrology, Phys. Rev. Lett. 96, 010401 (2006).

[38] D. V. Strekalov, A bundle of photons, please, Nat. Photonics 8 , 500 (2014)

[39] J.-W. Pan, Z.-B. Chen, C.-Y. Lu, H. Weinfurter, A. Zeilinger, and M. Żukowski, Multiphoton entanglement and interferometry, Rev. Mod. Phys. 84, 777 (2012).

[40] C. Sánchez Muñoz, E. del Valle, A. González Tudela, K. Müller, S. Lichtmannecker, M. Kaniber, C. Tejedor, J. J. Finley, and F. P. Laussy, Emitters of n-photon bundles, Nat. Photonics 8, 550 (2014).

[41] C. S. Muñoz, F. P. Laussy, C. Tejedor, and E. del Valle, Enhanced two-photon emission from a dressed biexciton, New J. Phys. 17, 123021 (2015). 
[42] Y. Chang, A. González-Tudela, C. Sánchez Muñoz, C. Navarrete-Benlloch, and T. Shi, Deterministic Down-Converter and Continuous Photon-Pair Source within the Bad-Cavity Limit, Phys. Rev. Lett. 117, 203602 (2016).

[43] E. Sánchez-Burillo, L. Martín-Moreno, J. J. García-Ripoll, and D. Zueco, Full two-photon down-conversion of a single photon, Phys. Rev. A 94, 053814 (2016).

[44] C. S. Muñoz, F. P. Laussy, E. del Valle, C. Tejedor, and A. González-Tudela, Filtering multiphoton emission from state-of-the-art cavity quantum electrodynamics, Optica 5, 14 (2018).

[45] X.-L. Dong and P.-B. Li, Multiphonon interactions between nitrogen-vacancy centers and nanomechanical resonators, Phys. Rev. A 100, 043825 (2019).

[46] Q. Bin, X.-Y. Lü, F. P. Laussy, F. Nori, and Y. Wu, n-Phonon Bundle Emission via the Stokes Process, Phys. Rev. Lett. 124, 053601 (2020).

[47] Y. Deng, T. Shi, and S. Yi, Motional n-phonon bundle states of a trapped atom with clock transitions, Photonics Res. 9, 1289 (2021).

[48] Q. Bin, Y. Wu, and X.-Y. Lü, Parity-Symmetry-Protected Multiphoton Bundle Emission, Phys. Rev. Lett. 127, 073602 (2021).

[49] V. Bouchiat, D. Vion, P. Joyez, D. Esteve, and M. H. Devoret, Quantum coherence with a single Cooper pair, Phys. Scr. T76, 165 (1998)

[50] Y. Makhlin, G. Scöhn, and A. Shnirman, Josephson-junction qubits with controlled couplings, Nature (London) 398, 305 (1999).

[51] Y. Nakamura, Yu. A. Pashkin, and J. S. Tsai, Coherent control of macroscopic quantum states in a single-Cooper-pair box, Nature (London) 398, 786 (1999).

[52] A. Blais, R.-S. Huang, A. Wallraff, S. M. Girvin, and R. J. Schoelkopf, Cavity quantum electrodynamics for su- perconducting electrical circuits: An architecture for quantum computation, Phys. Rev. A 69, 062320 (2004).

[53] D. Leibfried, R. Blatt, C. Monroe, and D. Wineland, Quantum dynamics of single trapped ions, Rev. Mod. Phys. 75, 281 (2003).

[54] C. Hamsen, K. N. Tolazzi, T. Wilk, and G. Rempe, Two-Photon Blockade in an Atom-Driven Cavity QED System, Phys. Rev. Lett. 118, 133604 (2017).

[55] A. Miranowicz, M. Paprzycka, Y. Liu, J. Bajer, and F. Nori, Two-photon and three-photon blockades in driven nonlinear systems, Phys. Rev. A 87, 023809 (2013).

[56] S. S. Shamailov, A. S. Parkins, M. J. Collett, and H. J. Carmichael, Multi-photon blockade and dressing of the dressed states, Opt. Commun. 283, 766 (2010).

[57] C. J. Zhu, Y. P. Yang, and G. S. Agarwal, Collective multiphoton blockade in cavity quantum electrodynamics, Phys. Rev. A 95, 063842 (2017).

[58] R. Huang, A. Miranowicz, J.-Q. Liao, F. Nori, and H. Jing, Nonreciprocal Photon Blockade, Phys. Rev. Lett. 121, 153601 (2018).

[59] Z. Kim, B. Suri, V. Zaretskey, S. Novikov, K. D. Osborn, A. Mizel, F. C. Wellstood, and B. S. Palmer, Decoupling a CooperPair Box to Enhance the Lifetime to $0.2 \mathrm{~ms}$, Phys. Rev. Lett. 106, 120501 (2011).

[60] S. Kono, K. Koshino, D. Lachance-Quirion, A. F. van Loo, Y Tabuchi, A. Noguchi, and Y. Nakamura, Breaking the trade-off between fast control and long lifetime of a superconducting qubit, Nat. Commun. 11, 3683 (2020).

[61] J. R. Johansson, P. D. Nation, and F. Nori, QuTiP: An opensource python framework for the dynamics of open quantum systems, Comput. Phys. Commun. 183, 1760 (2012).

[62] K. E. Cahill and R. J. Glauber, Ordered expansions in boson amplitude operators, Phys. Rev. 177, 1857 (1969). 